\title{
Braquiterapia endoluminal como tratamiento del carcinoma de esófago en estadio I
}

\author{
P. J. Prada, A. de la Rua, R. Rodríguez
}

El cáncer de esófago ocupa el quinto lugar entre los tumores malignos del hombre. Sin embargo, su incidencia presenta importantes variaciones geográficas, fruto de las diferencias existentes en la distribución de los factores ambientales y socioculturales implicados en su etiología. En nuestro medio, la incidencia es de 5 casos/100.000 habitantes/ año, con una afectación máxima entre los 50 y 70 años, siendo más frecuente en el hombre que en la mujer.

La mayoría de los pacientes presentan al diagnóstico un estadio avanzado, ya que éste se suele realizar cuando aparece la clínica, la cual se presenta cuando el tumor tiene una extensión ya considerable, momento en el que las posibilidades terapéuticas de curación son escasas. Por tanto el número de pacientes diagnosticados en estadio I', representan actualmente una minoría en nuestro medio, pero son ellos los que tienen las mayores posibilidades de curación, de ahí la importancia del diagnóstico precoz.

Cuando diagnosticamos a un paciente en estadio inicial de la enfermedad, necesitamos tener presente:

- Por un lado, que debemos conocer la profundidad de afectación de la pared esofágica, ya que este hecho constituye un importante factor pronóstico que se relaciona directamente con el control local y la supervivencia ${ }^{2,4}$. Además, el establecimiento de un minucioso estadiaje, va a ser también quien determine la opción terapéutica más eficaz y con menor número de complicaciones. Bajo este punto de vista, la ecografía endoluminal sería el medio diagnóstico que proporcionaría mayor certeza en la identificación de la afectación de la pared esofágica por el tumor y por tanto, el estadio ${ }^{5-7}$.

- Por otra parte, sabemos que el tratamiento estándar de los tumores en estadios iniciales, es la cirugía. Las grandes series quirúrgicas japonesas en tumores esofágicos superficiales presentan resultados de supervivencia a cinco años entre el 65 y el $90 \% 6$. Pero también no conviene olvidar que el tratamiento quirúrgico es de una agresividad

Servicio de Oncología Radioterápica Hospital Universitario Central Asturias (Oviedo) importante, con una mortalidad operatoria alrededor del $10 \%$ y perioperatoria del $17 \%{ }^{8}$, con un índice de complicaciones globales que puede exceder el 75\% (pulmonares, cardíacas, de la anastomosis, estenosis, infecciones). Además pueden existir problemas en la radicalidad, ya que un porcentaje no despreciable de pacientes presentan márgenes quirúrgicos afectados por el tumor después de la intervención. Esto, unido al hecho de que muchos de estos enfermos presentan una edad avanzada, con importantes problemas médicos asociados que hacen que un grupo no despreciable de pacientes sean considerados inoperables, ha hecho que se busquen otras soluciones de tratamiento alternativas.

Una de estas alternativas terapéuticas de intención curativa la constituye la radioterapia. Pero la radioterapia externa clásica no está exenta de problemas, y así por un lado sabemos que el tumor esofágico es dosis-dependiente, por lo que a mayor dosis de irradiación administrada mayores probabilidades de control tumoral, lo cual choca de frente con el hecho de las posibilidades reales de irradiación que podemos dar y que viene determinada por la tolerancia de los tejidos sanos de alrededor (médula, pulmón, corazón). Además, la irradiación externa presenta problemas con la reproducción diaria del tratamiento que puede conllevar errores de administración de la dosis en el lugar preciso.

Por todo ello hemos desarrollado la técnica de radioterapia intraluminal con HDR a nivel esofágico, siguiendo el Consenso Americano de braquiterapia esofágica9, ya que nos permite:

- Aumentar la dosis de irradiación a nivel tumoral;

- Disminuir las complicaciones;

- Evitar la irradiación de los tejidos sanos de alrededor;

- Precisión en la administración de la dosis en el mismo seno del tumor;

- Tratamiento más corto.

En nuestro Servicio de Oncología Radioterápica, han recibido tratamiento con una combinación de irradiación externa y HDR un total de 20 pacientes, de los cuales 10 fueron estadios I. Todos los pacientes tratados fueron varones, con una edad media de 67 años (47-78 años) y una mediana de 69 años, con diagnóstico histológico de tumores escamosos. En cuanto a las características del tumor, destacar que todos ellos asentaron en el tercio medio del esófago, fueron lesiones esofíticas; en siete casos la afectación 
de la circunferencia esofágica fue igual o inferior a la mitad de la misma y sólo en un caso la afectación fue completa.

Respecto al tratamiento, todos los pacientes recibieron irradiación externa a dosis de 45-50Gy sobre primario y cadenas de drenaje ganglionar, asociado a tratamiento citostático; la braquiterapia se administró entre la segunda y tercera semanas tras la irradiación externa y consistió, en la mayoría de los casos, en dos aplicaciones de 500cGY cada una de ellas separadas una semana; la dosis se administró a $1 \mathrm{~cm}$ de profundidad y la longitud media de tratamiento con braquiterapia fue de $7 \mathrm{~cm}(5-9 \mathrm{~cm})$.

La media de seguimiento para el grupo completo fue de 21 meses (1-65 meses) y la mediana de 12 meses.

La respuesta al tratamiento radiológico evaluada por esofagoscopia tras la irradiación externa y antes de tratamiento braquiterapia, fue macroscópicamente completa en ocho casos, parcial en un caso y no se especifica en otro.

En el grupo analizado, sólo un paciente presentó recaída a nivel del tumor primario, lo que supuso una supervivencia libre de recaída a nivel del esófago así como supervivencia libre de enfermedad del $86 \%$ evaluada a cinco años y cinco meses. Si bien es cierto que el número de pacientes con un seguimiento largo fue solamente de tres casos, también es cierto que el otro grupo de pacientes con seguimientos menores y que fueron exitus a consecuencia de otra enfermedad, se encontraban libres de recidiva tumoral y quizás lo más destacable, que todos ellos durante el tiempo que vivieron presentaron una deglución normal.

En cuanto a las complicaciones, destacar que durante el tratamiento se presentaron molestias esofágicas catalogadas como grado I-II de la RTOG, molestias que se controlaron con tratamiento médico. A largo plazo sólo destacar como complicación de mayor importancia, una estenosis en uno de los pacientes que obligó a dilataciones endoscópicas.

En la actualidad, hay un número considerable de publicaciones acerca de la utilización de braquiterapia en el cáncer de esófago en las que se destacan las ventajas de la utilización de dicha técnica.

Okawa y cols. ${ }^{10}$ han comparado los resultados de los grupos de pacientes, unos tratados con irradiación externa sólo y otros con una combinación irradiación externa y HDR, comunicando un control local superior para el grupo en el que se realizó tratamiento con radioterapia externa más braquiterapia endoluminal.

Nishikawa y cols. ${ }^{11,} 12$ han publicado no sólo un aumento del control local para los pacientes tratados con braquiterapia sino también un aumento de la supervivencia.

Nemoto y cols., 13 manifiestan que la utilización de HDR mejora la supervivencia y el control local de los tumores superficiales, presentando una supervivencia causa específica a los cinco años, para los tumores que afectan sólo a la mucosa, del $81 \%$ y el $64 \%$ para los que afectan la submucosa, con un control local de la enfermedad del $88 \%$ en los tumores confinados a la mucosa y el $77 \%$ para los afectan a la submucosa. El primero de los grupos no presentó metástasis, mientras que en el segundo grupo se produjo diseminación distal en el $16 \%$ de las pacientes.

Otros autores como Maingon, Nishimura, Arrellana, Gaspar y Fuwa refieren resultados semejantes ${ }^{14-18}$. Todos los grupos comunican un porcentaje de complicaciones aceptables ${ }^{19,20}$
Bajo estos conocimientos y la propia experiencia, proponemos como opción terapéutica la cirugía en estadios iniciales, con el siguiente esquema terapéutico:

- Tumores que afectan sólo a la mucosa. Braquiterapia endoluminal de Alta tasa dosis exclusiva.

- Tumores que afectan a la submucosa. Radioterapia externa más braquiterapia endoluminal de alta tasa dosis.

- Tumores con afectación mayor. Radioterapia externa más braquiterapia endoluminal de alta tasa de dosis más quimioterapia.

Este esquema de tratamiento presenta como ventaja añadida, que ante una posible recaída a nivel del tumor primario, es posible realizar cirugía de rescate, ya que las dosis irradiación externa estarían limitadas a dosis de $45 \mathrm{~Gy}$, que son las utilizadas en los tratamientos combinados de radiocirugía, y sabemos que las dosis que proporciona la aplicación endoluminal de braquiterapia es tan limitada a la zona de asiento tumoral, que no impide la realización de una cirugía de rescate ni añade más complicaciones a la misma.

\section{Bibliografía}

1. Sobin LH, Hermanek P, Hutter RVP. TNM classification of malignant tumors: A comparison between the new (1987) and old edition. Cancer 1988; 6:2310-14.

2. Akagi $Y$, Hirokawa $Y$, Kagemoto $M$, et al. Optimum fractionation for high-dose-rate endoesophageal brachytherapy following external irradiation of early stage esophageal cancer. Int J Radiat Oncol Biol Phys 1999; 43:525-30.

3. Nemoto K, Matsumoto Y, Yamakawa M, et al. Treatment of superficial esophageal cancer by external radiation therapy alone: Results of a muti-institutional experience. Int J Radiat Oncol Biol Phys 2000; 46:921-5.

4. Nabeya K, Nakata Y. Extent of resection and lymphadenectomy in early squamous cell esophageal cancer. Dis Esophagus 1997; 10:159-61.

5. Fukuda M, Hirata K, Natori H. Endoscopic ultrasonography of the esophagus. World J Surg 2000; 24:216-26.

6. Hasegawa N, Niwa Y, Arisawa T, et al. Preoperative staging of superficial esophageal carcinoma: Comparison of an ultrasound probe and standard endoscopic ultrasonography. Gastrointest Endosc 1996; 44:388-93.

7. Botet JF, Lightdaie $\mathrm{Cl}$, Zauber AG, et al. Preoperative staging of esophageal cancer: Comparison of endoscopic US and dynamic CT. Radiology 1991; 181:419-25.

8. Tsuti S, Moriguchi S, Morita M, et al. Multivariate analysis of postoperative complications after esofhageal resection. Ann Thorac Surg 1992; 53:1052.

9. Gaspar LE, Nag S, Herskovic A, et al. American Brachytherapy Society consensus guidelines for brachytherapy of esophageal cancer. Int J Radiat Oncol Biol Phys 1997; 38: 127-32.

10. Okawa T, Tanaka M, Kita M, et al. Radiotherapy for superficial esophageal cancer. Int J Radiat Oncol Biol Phys 1994; 30:95964.

11. Hishikawa $Y$, Kurisi K, Tanigushi $M$, et al. High dose rate brachytherapy for esophageal cancer. Int J Radiat Oncol Biol Phys 1991; 21:1133-5.

12. Hishikawa $Y$, Kurisu K, Taniguchi M, Kamikonya N, Miura T. 


\section{P. J. Prada y cols.}

High-dose-rate intraluminal brachytherapy for esophageal cancer: 10 years experience in Hyogo College of Medicine. Radiother Oncology 1991; 21:107-14.

13. Nemoto K, Yamada S, Harreyana M, et al. Radiation Therapy for superficial esophageal cancer: A comparison of radiotherapy methods. Int J Radiat Oncol Biol Phys 2001; 50:639-44.

14. Maingon $P, D^{\prime}$ Hombres $A$, Truc $G$, et al. High dose rate brachytherapy for superficial cancer of the esophagus. Int J Radiat Oncol Biol Phys 2000; 46:71-6.

15. Nishimura $Y$, Okuno $Y$, Ono $K$, et al. External beam radiation therapy with or without high-dose-rate intraluminal brachytherapy for patients with superficial esophageal carcinoma. Cancer 1999; 15:220-8.

16. Hareyama $M$, Nishio $M$, Kagami $Y$, et al. Intracavitary brachytherapy combined with externat beam irradiation for squamous cell carcinoma of the thoracic esophagus. Int J Radiat Oncol Biol Phys 1992; 24:235-40.
17. Gaspar L, Winter K, Kocha WI, et al. A phase I / II study of externat beam radiation, brachytherapy, and concurrent chemotherapy for patients with localized carcinoma of the esophagus (RTOG 9207). Final report. Cancer 2000; 88:988-95.

18. Fuwa N, Nomoto $Y$, Shouji $K$ et al. Therapeutic effects of simultaneous intraluminal irradiation and intraluminal hyperthermia on oesophageal carcinoma. The British Journal of Radiology 2001; 74:709-14.

19. Gaspar L, Chunlin Qian, Kocha WI, et al. A phase I / II study of externat beam radiation, brachytherapy, and concurrent chemotherapy in localized cancer of the esophagus (RTOG 9207): Preliminary toxicity report. Int J Radiat Oncol Biol Phys 1997; 37:593-9.

20. Sharma V, Agarwal J, Dinshaw K, et al. Late esophageal toxicity using a combination of beam radiation, intraluminal brachytherapy and 5-fluorouracil infusión in carcinoma of the esophagus. Diseases of the Esophagus 2000; 13:219-25. 